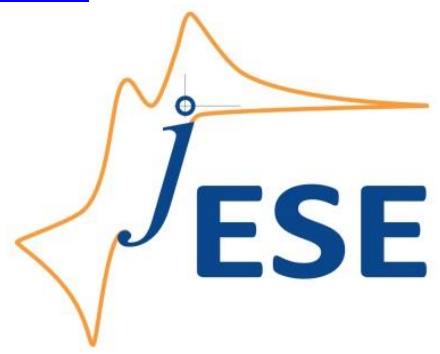

Open Access : : ISSN 1847-9286

www.jESE-online.org

Original scientific paper

\title{
Enhancement of the cerium oxide primer layers deposited on AA2024-T3 aircraft alloy by preliminary anodization
}

\author{
Stephan Kozhukharov ${ }^{1, \otimes}$, Christian Girginov ${ }^{2}$ \\ ${ }^{1}$ LAMAR - Laboratory, University of Chemical Technology and Metallurgy - Sofia, 8 "Kliment \\ Okhridsky" Blvd. 1756, Sofia, Bulgaria \\ ${ }^{2}$ Department of Chemical Sciences, University of Chemical Technology and Metallurgy - Sofia, \\ 8 "Kliment Okhridsky" Blvd. 1756, Sofia, Bulgaria
}

Corresponding authors e-mail: ${ }^{\bowtie}$ stephko1980@abv.bg; Tel.: +359899837282

Received: November 28, 2017; Revised: February 2, 2018; Accepted: February 8, 2018

\begin{abstract}
The possibility for combination between anodized aluminum oxide (AAO) and cerium oxide primer layer (CeOPL) for elaboration of efficient protective coatings for AA2024-T3 aircraft alloy is proposed in the present research. The combined AAO/CeOPL coating characterizations include electrochemical impedance spectroscopy (EIS) combined with linear voltammetry (LVA), for extended times (until 2520 hours) to a model corrosive medium (3.5\% NaCl). Topographical and cross-sectional (SEM and EDX) observations were performed in order to determine the AAO/CeOPL film thickness and composition. The AAO/CeOPL layer durability tests were confirmed by standard neutral salt spray (NSS). The data analysis from all the used measurement methods has undoubtedly shown that the presence of AAO film significantly improves the cerium oxide primer layer (CeOPL) protective properties and performance.
\end{abstract}

\section{Keywords}

AA2024-T3 alloy; anodization; Cerium oxide primer layers; Barrier ability; Durability

\section{Introduction}

Although the anodization process is well investigated for pure aluminum [1], its highly doped alloys reveal significant deviations from the expected behavior, due to their compositional and structural heterogeneity [2-9]. In the latter case, the intermetallic inclusions entirely predetermine the mechanism and kinetics of both the primer protective layer deposition and the corrosion processes. Thus, the method applied for preliminary surface treatment completely predetermines the chemical composition of the Al-oxide surface layer in sense of correlation between anhydrous aluminum oxide $\mathrm{Al}_{2} \mathrm{O}_{3}$ and crystalline boehmite $(\mathrm{AlOOH})$, the hydroxide and adsorbed water contents, etc. $[10,11]$. Besides, the surface oxide layer composition, thickness and properties are 
of crucial importance for the primer coating layer adhesion $[12,13]$. Thus, in a previous work it was demonstrated that the anodization in sulfuric acid enables the growth of rather thick anodized alumina oxide (AAO) layers [14], efficiently compensating the AA2024-T3 heterogeneity.

On the other hand, the cerium conversion coatings (CeCC), appear to be an efficient environmentally compliant chromium substitute $[15,16]$. However, it is widely accepted to treat the metallic substrate only by chemical or mechanical methods [14,16], prior to CeCC deposition. Consequently, the preliminary anodization prior to cerium oxide primer layer (CeOPL) deposition should have a remarkable beneficial effect on the performance of the obtained AAO/CeOPL conjunction. The obtained double layer coating systems should possess significantly extended durability and enhanced barrier properties, providing reliable and durable corrosion protective ability. The expected synergism between the AAO and CeOPL is not limited only to the additive effect between the AAO and CeOPL barrier properties but it also includes CeOPL adhesion enhancement, due to the AAO porosity, providing enlarged contact surface area. Furthermore, the porous thick AAO provide uniform Ce-oxide distribution and thicker CeOPL layers. In this case, according to various authors the CeOPL layers should possess significant adhesion, predetermined by the Al-O-Ce covalent bonds composing the interfaces of the entire Al/AAO/CeOPL conjunction [17-21]. Finally, it is worth mentioning that the anodization procedure is easily controllable and provides AAO parameters variation via relatively simple regime parameter control. As a conclusion of all the above-mentioned facts, the combination between AAO and CeOPL should provide rather extended corrosion protective capabilities, being really successful strategy for efficient corrosion protection of various aluminum and other metallic substrates.

The aim of the present study is to determine the impact of the preliminary anodization process duration on the subsequent cerium oxide layer (CeOPL) properties and behavior in a model corrosive medium.

\section{Experimental}

Four sets of three AA2024-T3 plates were submitted to a sequence of procedures for repeatable $\mathrm{AAO} / \mathrm{CeOPL}$ bi-layer primer coating deposition. Initially, each sample was etched in $50 \mathrm{~g} / \mathrm{l} \mathrm{NaOH}$ alkaline medium at $60{ }^{\circ} \mathrm{C}$, and desmutted in diluted $\mathrm{HNO}_{3}(1: 1 \mathrm{v} / \mathrm{v})$. Each procedure was performed for $2 \mathrm{~min}$, with subsequent vigorous washing with tap water for at least $2 \mathrm{~min}$. Afterwards, the preliminary treated specimens were additively cleaned by distilled water and mounted in a two-electrode cell with similar design as described in a previous work [2]. This cell ensured uniform anodization of circular area of a $4 \mathrm{~cm}^{2}$. The cathode was platinum mesh and the electrolyte was composed of $15 \% w t . \mathrm{H}_{2} \mathrm{SO}_{4}$ solution. The anodization procedures were performed in galvanostatic regime $\left(15 \mathrm{~mA} \mathrm{~cm}^{-2}\right)$, in triplicate for 12,24 , or $48 \mathrm{~min}$ at room temperature and continuous stirring at $120 \mathrm{rpm}$.

This procedure was followed by spontaneous CeOPL deposition for $4 \mathrm{~min}$ at $60{ }^{\circ} \mathrm{C}$, in cerium containing aqueous mixture. It was preliminary prepared by mixing of $16.8 \mathrm{~g}$. of $98 \%$ anhydrous diammonium pentanitrocerate $\left(\left(\mathrm{NH}_{4}\right)_{2} \mathrm{Ce}\left(\mathrm{NO}_{3}\right)_{5}\right.$, Fluka Chemica -Switzerland), $20.0 \mathrm{~g}$. NaCl (SigmaAldrich) and $5.0 \mathrm{ml}$. of $30 \% \mathrm{H}_{2} \mathrm{O}_{2}$ (Sigma-Aldrich) in a volumetric flask (1000 ml.). This stock solution was used for all the depositions done. The specimen assignations are shown in Table 1.

Electrochemical studies were performed periodically (once a week), during continuous exposure to the $3.5 \% \mathrm{NaCl}$ model corrosive medium. The measurements were performed using electrochemical impedance spectroscopy (EIS), from $10 \mathrm{kHz}$ to $10 \mathrm{mHz}$, distributed in 7 frequency steps per decade. 
Table 1. Assignations of the investigated specimens

\begin{tabular}{ccccc}
\hline \multicolumn{5}{c}{ CeOPL coated sets of samples } \\
\hline Samples No & References & Anodized for 12 min & Anodized for 24 min. & Anodized for 48 min \\
\hline \hline 1 & $1 S 0$ & $1 S 12$ & $1 S 24$ & $1 S 48$ \\
2 & $2 S 0$ & $2 S 12$ & $2 S 24$ & $2 S 48$ \\
3 & $3 S 0$ & $3 S 12$ & $3 S 24$ & $3 S 48$ \\
\hline
\end{tabular}

The AC signal amplitude was 15 to $45 \mathrm{mV}$, according to open circuit potential (OCP). Its amplitude was determined as the lowest possible value, enabling the acquisition of readable impedance spectrum. It is worth noting that when the amplitude was too high, inductance appeared, indicating electrode polarization. The EIS spectra, as well as all the rest electrochemical measurements were recorded versus $(\mathrm{Ag} / \mathrm{AgCl} 3 \mathrm{M} \mathrm{KCl})$ reference electrode. The EIS spectra acquisitions were followed by linear voltammetry. The voltammetric measurements were performed in subsequence of cathodic (from 30 to $-600 \mathrm{mV}$ ) and anodic (from -30 to $600 \mathrm{mV}$ ) ranges, both at $10 \mathrm{mV}$ $\mathrm{s}^{-1}$ scan rate. These measurements were performed in similar cells, but without stirring. Besides, the exposed area was lower $\left(2 \mathrm{~cm}^{2}\right)$, in order to avoid undesirable edge effect phenomena.

The resulting film morphology and thickness was determined by scanning electron microscopy, (SEM) performed by TESCAN, SEM/FIB LYRA I XMU working at $30 \mathrm{kV}$. The SEM observations were combined by elemental analysis, with energy dispersion spectroscopy (EDX) using energy dispersion spectrometer Quantax 200 of BRUKER.

In order to determine the coating performance in severe service conditions, neutral salt spray (NSS) test procedure was performed, according to ISO 9227 standard. This procedure was performed on nine square samples of the same alloy, but with larger dimensions (60×60 mm). Prior to the test procedures, AAO/CeOPL double layered coatings were deposited following the procedures described above. The surface areas of the double coatings were with $40 \mathrm{~mm}$ of diameter, (providing $12.50 \mathrm{~cm}^{2}$ ). The test procedure was performed for one complete week cycle (168 h) in VSN 1000 humidity chamber (Heraeus-Vötsch GmbH, Germany) by spraying of $5 \% \mathrm{NaCl}$ aqueous solution with $\mathrm{pH}$ range between 6.5 and 7.2. The samples were positioned at $20 \pm 5^{\circ}$ of slope. The in-chamber humidity was high enough to enable water to condense of at least 1 to $2 \mathrm{ml} \mathrm{h}^{-1}$. The corrosion impact rate was observed visually and estimated as a number of localized corrosion damaged domains from the entire exposed surface, according to the Bulgarian National Standard, BDS 15258-81, method C.

\section{Results and discussion}

Performance in a model corrosive medium - The performance of the obtained AAO/CeOPL double layers was determined by electrochemical measurements executed once of week during extended exposition of the investigated samples to $3.5 \% \mathrm{NaCl}$ model corrosive medium. This approach enabled determination of the corrosion protective properties of the obtained oxide bilayers, conditionally divided into: barrier ability and durability [22].

Barrier ability - This property is actually the capability of any coating to obstruct the corrosive species access to the metallic surface. It can be evaluated by the electrical resistance increase, determined by electrochemical measurements (EIS and LVA), performed at the initial exposition period. Although the initial measurements were performed at the $24^{\text {th }}$ hour of exposure to the $\mathrm{NaCl}$ solution, it was preferred to represent the results acquired after 168 hours of exposition. After one week of exposure, the reference samples, (prepared by CeOPL deposition without preliminary anodization) were obviously affected by pitting corrosion unlike the double layered specimens. The average EIS spectra, acquired after 168 hours of exposure are shown in Fig. 1. 

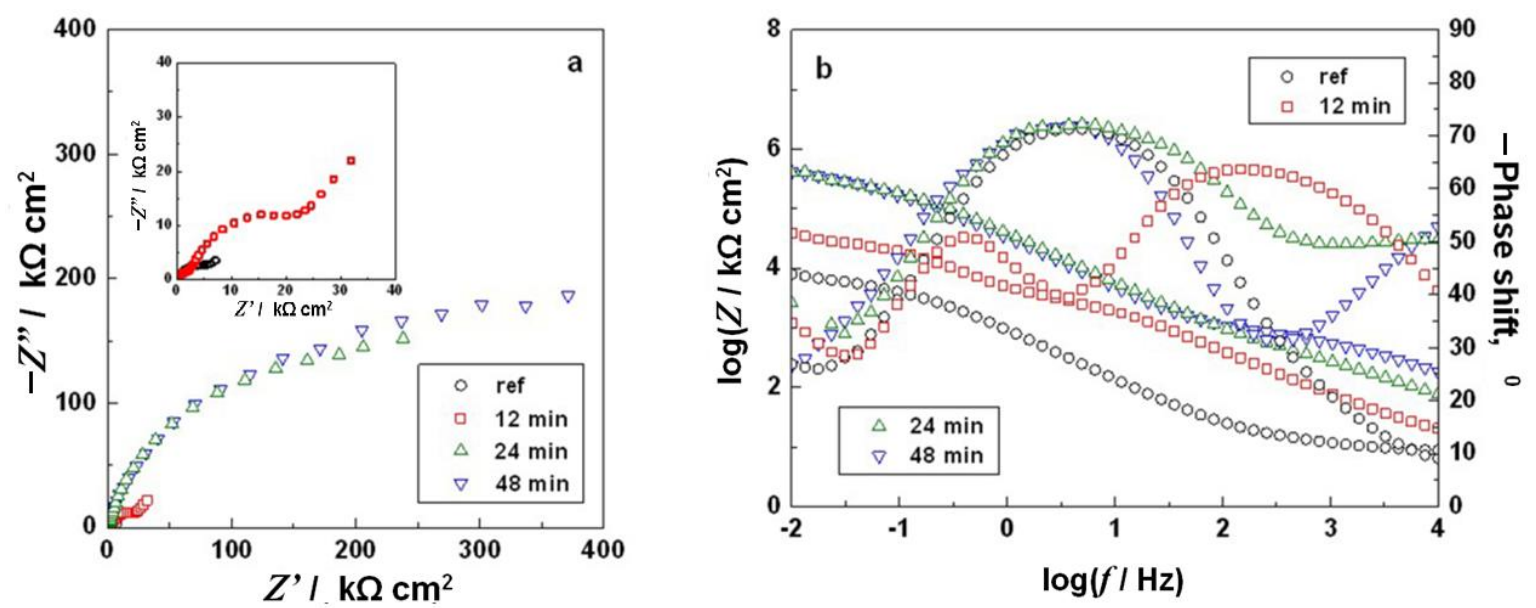

Figure 1. EIS spectra acquired after 168 hours of exposure for the investigated specimens for CeOPL deposited coatings after $0,12,24$ and 48 minutes of preliminary anodization.

As can be seen from Fig 1, the average spectra obtained for the respective sample sets are rather distinguishable among themselves.

The already corroded references ( 0 minutes of anodization) show simple spectra. Their Nyquist plots consist of slightly depressed semi-circles and Warburg diffusion tails (i.e. sloped straight lines). Besides, the phase shift/frequency lines in the Bode plots showed only one, very narrow minimum at about $10 \mathrm{~Hz}$.

The maxima in the spectra of the combined coating layers were wide (due to the overlapping of at least two peaks). Obviously, the appearance of additional peak is a contribution of the AAO formed during the anodization. This supplemental layer contributes for the increase of the total impedance logarithm $(\log |Z|)$ values, registered at $10 \mathrm{mHz}$, as well. These values increased from $10^{4} \Omega \mathrm{cm}^{2}$ for the references to almost $10^{6} \Omega \mathrm{cm}^{2}$ for the combined coatings.

The impedance spectra acquired after 168 hours of exposure were fitted to two different equivalent circuits (Fig. 2a, and b).

(a)

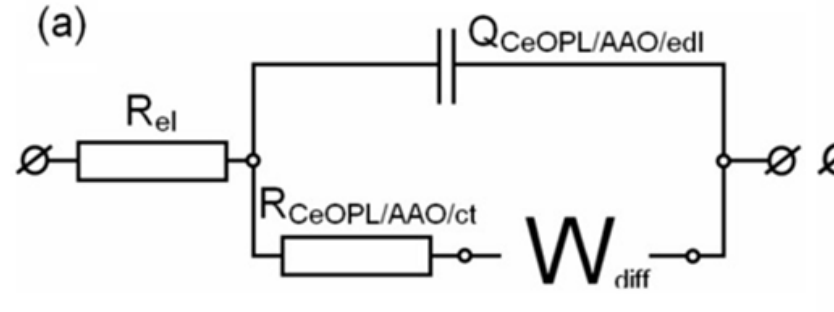

(b)

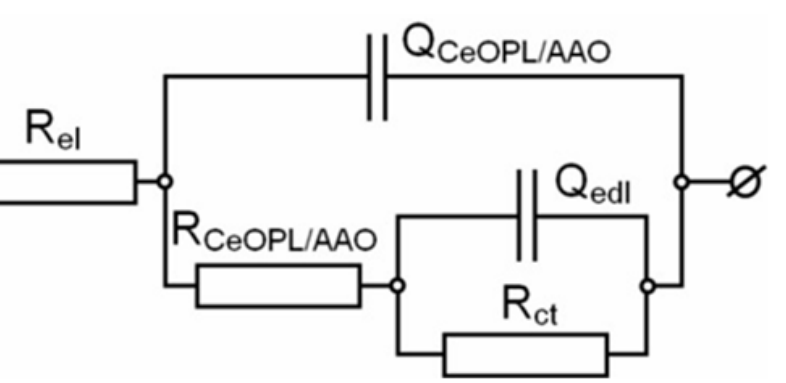

Figure 2. Equivalent circuits used for EIS-data fitting

$\mathrm{R}_{e l}-3.5 \% \mathrm{NaCl}$ electrolyte resistance; Q $\mathrm{Q}_{\mathrm{CeOP} / \mathrm{AAO} / \text { edl }}$ - Total CPE of the CeOPL, AAO, and the metal/electrolyte electric

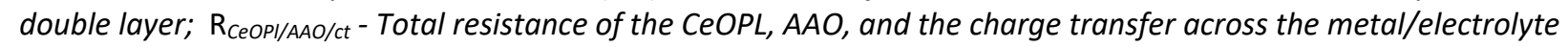
interface; $\mathrm{W}_{\text {diff }}$ - Warburg element for description of the diffusion of the corrosive species across the CeOPL defects;

$Q_{C e O P I / A A O}-C P E$ of the AAO/CeOPL interface; $R_{A A O / C e O P I}$ - Resistance of the AAO/CeOPL interface; $Q_{\text {edl }}$ - CPE of the

electyric double layer the metal/electrolyte interface; $\mathrm{R}_{c t}$ - charge transfer across the metal/electrolyte interface

Thus, the spectra of the corroded reference samples have simple shapes, corresponding to only one RQ unit (parallel resistance and constant phase element circuit), which includes a Warburg diffusion element, related to the diffusion limitations against the access of corrosive species towards the metallic surface, across the surface layer defects. All the rest specimens (with additional $A A O$ underlayer) revealed two consecutive $R Q$ units. 
Each one of the acquired impedance spectra, obtained after 168 hours of exposure was submitted to numerical fitting analysis by the equivalent circuits (Fig. 2). The obtained data for the impedance spectra elements are shown in Tables 2 and 3.

Table 2. Fitting results of the EIS spectra recorded after 168 hours for the referent CeOPL coated specimens

\begin{tabular}{ccccc}
\hline Element & Unit & Sample 1S0 & Sample 2S0 & Sample 3S0 \\
\hline$R_{\mathrm{el}}$ & $\Omega \mathrm{cm}^{2}$ & $19.44 \pm 0.59$ & $19.18 \pm 0.49$ & $20.02 \pm 0.59$ \\
$Q_{\text {coat+oxy+ct }}$ & $\mathrm{s}^{\mathrm{n}} \Omega^{-1} \mathrm{~cm}^{-2}$ & $0.76 \pm 0.03 \times 10^{-4}$ & $1.19 \pm 0.04 \times 10^{-4}$ & $1.12 \pm 0.05 \times 10^{-4}$ \\
$n$ & - & $0.83 \pm 0.95$ & $0.82 \pm 0.91$ & $0.81 \pm 0.01$ \\
$R_{\text {coat+oxy+edl }}$ & $\Omega \mathrm{cm}^{2}$ & $17.98 \pm 1.40 \times 10^{3}$ & $12.56 \pm 0.87 \times 10^{3}$ & $7.68 \pm 0.46 \times 10^{3}$ \\
$W_{\text {diff }}$ & $\mathrm{S}^{0.5} \Omega^{-1} \mathrm{~cm}^{-2}$ & $3.68 \pm 0.68 \times 10^{-4}$ & $6.50 \pm 1.30 \times 10^{-4}$ & $12.70 \pm 0.29 \times 10^{-4}$ \\
\hline
\end{tabular}

The numerical data for the referent samples (Table 2) show relatively low deviations. These specimens, with clear corrosion features (i.e. multitudes of pits) have shown total admittance between $0.29 \times 10^{-4}$ and $1.12 \times 10^{-4} \mathrm{~s}^{\mathrm{n}} \Omega^{-1} \mathrm{~cm}^{-2}$ and ohmic resistance between 7.68 and $19.98 \mathrm{k} \Omega \mathrm{cm}^{2}$. These values are in the typical range for corroded bare AA2024-T3 alloy, established in previous studies [23-25]. Besides, the data fitting for these specimens required addition of Warburg element. This fact is an evidence of free diffusion of corrosive species across both the native Al-oxide and CeOPL layers. Both these findings indicated that the directly deposited CeOPL layer does not possess any protective activity after 168 hours of exposure to $3.5 \% \mathrm{NaCl}$ model corrosive medium. That was the reason for termination of the exposure tests after this period.

On the other hand, the data results for all the CeOPL coated specimens after anodization were more appropriate for fitting to the other equivalent circuit (Fig. 2b), composed by two-time constants. It should be noted that these time constants are not related to the interfaces between the AAO and CeOPL layers, but rather these correspond to the interfaces between the AAO/CeOPL conjunction, the AA2024-T3 metallic base and the aqueous $\mathrm{NaCl}$ electrolyte. Probably, this conjunction is composed by covalent Ce-O-Al bonds, suggesting that there is not clear interface between the AAO and CeOPL layers. Besides, both AAO and CeOPL layers are composed by metallic oxides with apparent dielectric properties.

Due to these reasons, the equivalent circuit used for the reference specimens (Fig. 2a) was inappropriate for EIS data result fitting of the spectra acquired for the preliminary anodized AA2024-T3 samples. The second equivalent circuit (Fig. 2b) has shown to be more suitable and the fitting results are shown in Table 3.

The data acquired by the EIS spectra fitting to the equivalent circuit (Fig 2b) possess different deviations rates among the results for each sample. Thus, the CeOPL specimens coated, after only $12 \mathrm{~min}$ of anodization possess remarkable deviations, in the range of entire orders of magnitude. This fact indicates that this anodization time is obviously insufficient for creation of thick enough AAO films with uniform coverage. Besides, probably the randomly distributed intermetallic inclusions of the alloy substrate interrupt this film.

The comparison between the charge transfer resistance $\left(R_{\mathrm{ct}}\right)$ data in Table 3 with the combined resistance $\left(R_{\text {coat+oxy+edl }}\right)$, in Table 2 show obvious barrier ability elevation after the preliminary anodization. Consequently, the observed barrier ability improvement after anodization is an indication for the beneficial role of the preliminary anodization procedure on CeOPL performance.

Following the concept for island growth of Ce oxide layers [26], it should be assumed that the CeOPL formation begins from exactly these intermetallics. Besides, the most active CeOPL growth initiation centers should be the copper rich S-phase and $\theta$-phase inclusions [3], according to the following reactions $[3,26]$ : 
Table 3. Fitting results of the EIS spectra acquired after 168 hours of exposure for the anodized, CeOPL coated specimens

\begin{tabular}{|c|c|c|c|c|}
\hline \multicolumn{5}{|c|}{12 minutes of anodization } \\
\hline Element & Unit & Sample 1S12 & Sample 2S12 & Sample 3 S12 \\
\hline$R_{\mathrm{el}}$ & $\Omega \mathrm{cm}^{2}$ & $15.36 \pm 0.21$ & $22.78 \pm 2.54$ & $33.06 \pm 8.95$ \\
\hline$Q_{\text {coat+oxy }}$ & $\mathrm{s}^{\mathrm{n}} \Omega^{-1} \mathrm{~cm}^{-2}$ & $4.35 \pm 0.06 \times 10^{-5}$ & $0.79 \pm 0.10 \times 10^{-5}$ & $0.15 \pm 0.05 \times 10^{-5}$ \\
\hline$n$ & & $0.78 \pm 0.002$ & $0.77 \pm 0.02$ & $0.68 \pm 0.003$ \\
\hline$R_{\text {coat+oxy }}$ & $\Omega . \mathrm{cm}^{2}$ & $23.71 \pm 0.96 \times 10^{3}$ & $6.72 \pm 1.15 \times 10^{3}$ & $7.68 \pm 0.34 \times 10^{3}$ \\
\hline$Q_{\text {edl }}$ & $\mathrm{s}^{\mathrm{n}} \Omega^{-1} \mathrm{~cm}^{-2}$ & $30.92 \pm 5.18 \times 10^{-5}$ & $2.89 \pm 0.55 \times 10^{-5}$ & $0.05 \pm 0.08 \times 10^{-5}$ \\
\hline$n$ & & $0.75 \pm 0.08$ & $0.67 \pm 0.04$ & $0.95 \pm 0.01$ \\
\hline$R_{\mathrm{ct}}$ & $\Omega \mathrm{cm}^{2}$ & $2.42 \pm 0.24 \times 10^{5}$ & $0.91 \pm 0.08 \times 10^{5}$ & $78.40 \pm 5.42 \times 10^{5}$ \\
\hline \multicolumn{5}{|c|}{24 minutes of anodization } \\
\hline Element & Unit & Sample 1S24 & Sample 2S24 & Sample 3S24 \\
\hline$R_{\mathrm{el}}$ & $\Omega \mathrm{cm}^{2}$ & $16.46 \pm 1.69$ & $45.86 \pm 6.28$ & $17.28 \pm 1.73$ \\
\hline$Q_{\text {coat+oxy }}$ & $\mathrm{s}^{\mathrm{n}} \Omega^{-1} \mathrm{~cm}^{-2}$ & $2.52 \pm 0.48 \times 10^{-6}$ & $1.78 \pm 0.32 \times 10^{-6}$ & $0.90 \pm 0.09 \times 10^{-6}$ \\
\hline$n$ & & $0.71 \pm 0.01$ & $0.76 \pm 0.02$ & $0.71 \pm 0.02$ \\
\hline$R_{\text {coat+oxy }}$ & $\Omega \mathrm{cm}^{2}$ & $2.39 \pm 0.44 \times 10^{3}$ & $1.36 \pm 0.20 \times 10^{3}$ & $4.62 \pm 0.23 \times 10^{3}$ \\
\hline$Q_{\text {edl }}$ & $\mathrm{s}^{\mathrm{n}} \Omega^{-1} \mathrm{~cm}^{-2}$ & $1.31 \pm 0.42 \times 10^{-6}$ & $1.15 \pm 0.24 \times 10^{-6}$ & $0.88 \pm 0.08 \times 10^{-6}$ \\
\hline $\mathrm{n}$ & & $0.86 \pm 0.04$ & $0.87 \pm 0.03$ & $0.94 \pm 0.02$ \\
\hline$R_{\mathrm{ct}}$ & $\Omega \mathrm{cm}^{2}$ & $4.91 \pm 0.19 \times 10^{5}$ & $7.58 \pm 0.31 \times 10^{5}$ & $30.64 \pm 1.32 \times 10^{5}$ \\
\hline \multicolumn{5}{|c|}{48 minutes of anodization } \\
\hline Element & Unit & Sample 1S48 & Sample $\mathbf{2 S 4 8}$ & Sample 3548 \\
\hline$R_{\mathrm{el}}$ & $\Omega . \mathrm{cm}^{2}$ & $27.44 \pm 6.91$ & $25.82 \pm 12.12$ & $71.60 \pm 17.95$ \\
\hline$Q_{\text {coat+oxy }}$ & $\mathrm{s}^{\mathrm{n}} \Omega^{-1} \mathrm{~cm}^{-2}$ & $6.52 \pm 0.02 \times 10^{-6}$ & $2.43 \pm 0.09 \times 10^{-6}$ & $0.96 \pm 0.07 \times 10^{-6}$ \\
\hline$n$ & & $0.58 \pm 0.003$ & $0.63 \pm 0.004$ & $0.67 \pm 0.008$ \\
\hline$R_{\text {coat }+ \text { oxy }}$ & $\Omega . \mathrm{cm}^{2}$ & $3.74 \pm 0.18 \times 10^{3}$ & $2.60 \pm 0.06 \times 10^{3}$ & $5.74 \pm 0.14 \times 10^{3}$ \\
\hline$Q_{\text {edl }}$ & $\mathrm{s}^{\mathrm{n}} \Omega^{-1} \mathrm{~cm}^{-2}$ & $0.90 \pm 0.10 \times 10^{-6}$ & $1.36 \pm 0.07 \times 10^{-6}$ & $1.93 \pm 0.07 \times 10^{-6}$ \\
\hline$n$ & & $0.97 \pm 0.02$ & $0.96 \pm 0.009$ & $0.94 \pm 0.009$ \\
\hline$R_{\mathrm{ct}}$ & $\Omega \mathrm{cm}^{2}$ & $2.70 \pm 0.06 \times 10^{5}$ & $11.16 \pm 0.31 \times 10^{5}$ & $111.20 \pm 1.22 \times 10^{5}$ \\
\hline
\end{tabular}

Oxygen reduction:

$\mathrm{O}_{2}+2 \mathrm{H}_{2} \mathrm{O}+4 \mathrm{e}^{-} \rightarrow 4 \mathrm{OH}^{-}$

Formation of intermediated complex species:

$2 \mathrm{Ce}^{3+}$ aq. $+\mathrm{H}_{2} \mathrm{O}_{2}+2 \mathrm{OH}-\rightarrow \mathrm{Ce}(\mathrm{OH})_{2}{ }^{2+}$ aq.

Reaction of the obtained complex ions with hydroxyl ions near to the metallic surface:

$\mathrm{Ce}(\mathrm{OH})_{2}{ }^{2+}{ }_{\text {aq. }}+2 \mathrm{OH}^{-} \rightarrow \mathrm{Ce}(\mathrm{OH})_{4}$

Subsequent conversion of the cerium hydroxides to the respective oxides:

$\mathrm{Ce}(\mathrm{OH})_{4} \rightarrow \mathrm{CeO}_{2}+2 \mathrm{H}_{2} \mathrm{O}$

According to the authors, these reactions proceed accompanied by the following supplemental reaction of direct interaction of the $\mathrm{Ce}^{3+}$ with the hydroxyl ions:

$$
\mathrm{Ce}^{3+}+3 \mathrm{OH}^{-} \rightarrow \mathrm{Ce}(\mathrm{OH})_{3}
$$

However, all this chain of consecutive reactions is strongly dependent on the available anodic area, composed by the Al-matrix under the oxide layer defects, since it provides the electrons necessary for the oxygen reduction and the resulting in $\mathrm{OH}$ - ion generation. Namely, the predominant hydroxyl ion generation (i.e. alkalization) near these intermetallics causes $\mathrm{Ce}(\mathrm{OH})_{3}$ and/or $\mathrm{Ce}(\mathrm{OH})_{4}$ precipitation. Consequently, the oxygen reduction cathodic reaction rate on the intermetallics is strongly dependent on the anodic Al-dissolution (Reaction 6):

$$
\mathrm{Al}^{0} \rightarrow \mathrm{Al}^{3+}+3 \mathrm{e}^{-}
$$

Applying this model for the CeOPL deposited AA2024-T3 samples after anodization, it can be anticipated that the AAO film hinders the CeOPL formation, because it covers the Al-matrix anodic 
area, necessary to supply electrons for the cathodic $\mathrm{OH}$-generation reactions, and the resulting Ce-hydroxide precipitation.

Nevertheless, this concept is in contradiction with the data in Table 3. All the acquired resistance data (i.e. $R_{\text {coat+oxy }}$ and $R_{\mathrm{ct}}$ ) reveal remarkably distinguishable dissipations among the specimens of each group (e.g. 12, 24 and $48 \mathrm{~min}$ of anodization). Simultaneously, the result deviations possess very similar deviation rates and orders of magnitude among the respective sample groups. Thus, no clear trend of these values was observed, although the obvious oxide layer thickness increases within the anodization procedure continuation, established in a previous work [27]. These facts provide undoubted evidence for the low oxide layer density due to its probably porous structure. In addition, the difference between the equivalent circuits suitable for the EIS-data fitting of the references and the anodized specimens reveal completely different mechanism of CeOPL deposition after AAO formation. Following the concepts of Arenas and Damborenea [28], it can be assumed that the AAO/CeOPL conjunction is alumina layer with partial substitution by Ce-ions. Obviously, the CeOPL layers deposited on thicker AAO layer, formed at extended anodization times possess better coverage. Indeed, Conde et al. [29] remark that the CeOPL layer formation is based on chemisorption processes on the superficial hydrated oxide layer of the aluminum, as follows:

$$
\begin{aligned}
& -\mathrm{Al}-\mathrm{OH}^{2+} \leftrightarrow-\mathrm{AlOH}+\mathrm{H}+ \\
& -\mathrm{Al}-\mathrm{OH}+\mathrm{Ce}^{3+} \leftrightarrow[-\mathrm{Al}-\mathrm{O} \cdots \mathrm{Ce}(\mathrm{III})]^{2+}+2 \mathrm{H}^{+}
\end{aligned}
$$

Consequently, CeOPL deposition mechanism alteration occurs on the more completely hydrated AAO layers, occupying the entire metallic surface. Thus, the CeOPL deposition follows the mechanism, proposed by Conde et al. [29], described by reactions (7) and (8).

These trends of barrier ability enhancement by the preliminary anodization have been evinced by the concordance of the results acquired by both the EIS data analysis and the polarization curves, described below. Fig. 3 illustrates the general trends (of current densities decrease, combined by corrosion potential ( $\left.E_{\text {corr }}\right)$ shift in positive direction) for the polarization curves recorded after 168 hours of exposure.
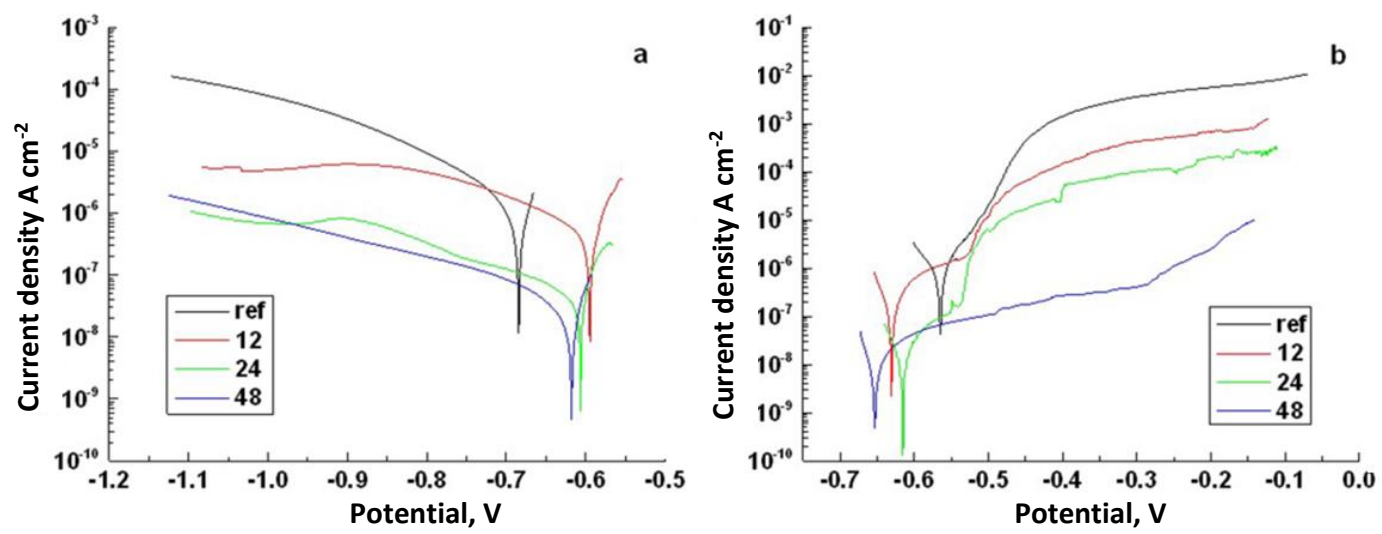

Figure 3. Cathodic (a) and anodic (b) polarization cures recorded after 168 hours of exposure

The polarization curves have been submitted to further Tafel plot analysis for determination of $E_{\text {corr }}$ and polarization resistance $\left(R_{\mathrm{p}}\right)$. The numerical data of the obtained results for all the investigated specimens are represented in Table 4. Some trends can be seen from the inferred, regardless the considerable result dissipation. The largest $E_{\text {corr }}$ difference between the cathodic and anodic curves belongs to the reference samples, showing that the relaxation time of about hours is not sufficient for $E_{\text {corr }}$ value restoration, after the cathodic curve acquisition. Unexpec- 
tedly, the $E_{\text {corr }}$ values of the anodic curves are with about $100 \mathrm{mV}$ more positive, although cathodic polarization of the specimens. Thus, during the cathodic curve acquisition, the investigated specimens (being negatively charged) attract cations from the model corrosive medium, like $\mathrm{Na}^{+}$ and $\mathrm{H}_{3} \mathrm{O}^{+}$, shifting $E_{\text {corr }}$ in positive direction. This assumption is additionally confirmed by the occurrence of Warburg diffusion elements, commented in the previous section.

Table 4. Tafel plot analysis of the obtained results from the polarization cures, recorded after 168 hours of exposure

\begin{tabular}{cccccccccccccc}
\hline \multicolumn{1}{c}{ Cathodic } & \multicolumn{1}{c}{ Anodic } \\
\hline & \multicolumn{1}{c}{$E_{\text {corr }} / \mathrm{V}$ vs. Ag/AgCl } & \multicolumn{3}{c}{$R_{\mathrm{p}} / \mathrm{k} \Omega \mathrm{cm}^{2}$} & \multicolumn{1}{c}{$E_{\text {corr }} / \mathrm{V}$ vs. Ag/AgCl } & \multicolumn{2}{c}{$R_{\mathrm{p}} / \mathrm{k} \Omega \mathrm{cm}^{2}$} \\
\hline Ref. & -0.658 & -0.685 & -0.718 & 17.8 & 14.0 & 8.6 & -0.557 & -0.565 & -0.536 & 18.4 & 10.0 & 8.4 \\
$12 \mathrm{~min}$ & -0.628 & -0.595 & -0.611 & 300.0 & 240.0 & 160.8 & -0.589 & -0.631 & -0.608 & 248.6 & 220.0 & 170.0 \\
$24 \mathrm{~min}$ & -0.633 & -0.607 & -0.773 & 480.0 & 280.0 & 196.0 & -0.571 & -0.615 & -0.685 & 600.80 & 380.0 & 214.0 \\
$48 \mathrm{~min}$ & -0.673 & -0.617 & -0.639 & 1980.0 & 3800.0 & 2200.0 & -0.676 & -0.653 & -0.614 & 2345.0 & 3600.0 & 2400.0 \\
\hline
\end{tabular}

The cathodic curves acquired from the reference specimens approximated straight lines, possessing indistinguishable curvature. Their slopes are probably result of electrolyte ohmic drop, and diffusion hindering in the electrolyte bulk. All other polarization curves obtained from the preliminary anodized samples had more similar $E_{\text {corr }}$ values, with deviations of about 35 to $40 \mathrm{mV}$. Besides, their cathodic curves stay at lower current densities compared to those of the reference samples. Consequently, the AAO/CeOPL conjunctions possess much better insulating properties, compared to the reference CeOPL monolayers. This inference is additionally confirmed by the relatively higher $R_{\mathrm{p}}$ values of the combined layer coatings, although the considerable value deviations among the samples. In all cases, the CeOPL coated specimens after 48 min of anodization possess at least an order of magnitude higher $R_{\mathrm{p}}$ values, compared to those, anodized for only $12 \mathrm{~min}$. The specimens treated for $24 \mathrm{~min}$ have intermediate position, being similar either to the former, or to the latter specimens. The higher barrier ability of the AAO/CeOPL layers suggests extended durability, and this fact was the reason to continue the experiments, related to the sample exposure to the model corrosive medium.

Durability - This property was evaluated by further extension of the sample exposure until corrosion pits appear, combined with regular EIS and LVA measurements, according to the regimes, described in the experimental section. Since this approach has provided a huge number of data collections, only the final measurements will be commented in detail. The sample durability of the specimens increases progressively with the anodization process duration (and the

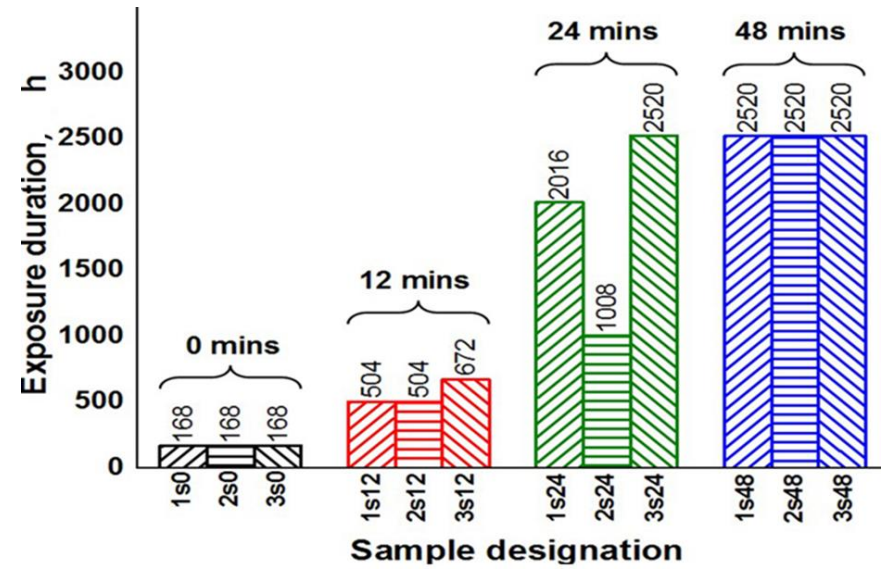

Figure 4. Durability diagram of the investigated specimens AAO thickness, respectively). Thus, the reference CeOPL monolayers have already failed until the $168^{\text {th }}$ hour of exposure, whereas the AAO/CeOPL layers, obtained by $48 \mathrm{~min}$ of anodization have resisted whole 2,520 hours in the model corrosive medium (Fig. 4)

The EIS spectra recorded for the final exposure times were appropriated for fitting to the equivalent circuit shown in Fig. 2b. Since the CeOPL coated specimens, after 24 min of anodization fall between the anodized for 12 and 48 
min, their results were not submitted to fitting. Furthermore, the significant difference of the exposure times registered for these samplers (e.g. the anodized for $24 \mathrm{~min}$ ) should lead to remarkable deviation among the respective EIS data fitting. The results, obtained from the final EIS spectra fitting are represented in Table 5.

It is worth mentioning, that at the final measurements (Fig. 4) all the preliminary anodized specimens possessed superior charge transfer resistance $\left(R_{\mathrm{ct}}\right)$ values (Table 5$)$, compared to those of the references after 168 hours of exposure (Table 2). Although the fact that the equivalent circuit was appropriate for data fitting in both cases of the specimens, preliminary anodized for 12 and $48 \mathrm{~min}$, their spectra had rather distinguishable shapes (Fig. 5). Since the specimens anodized for 24 min have shown great deviations among themselves, their spectra are not shown.
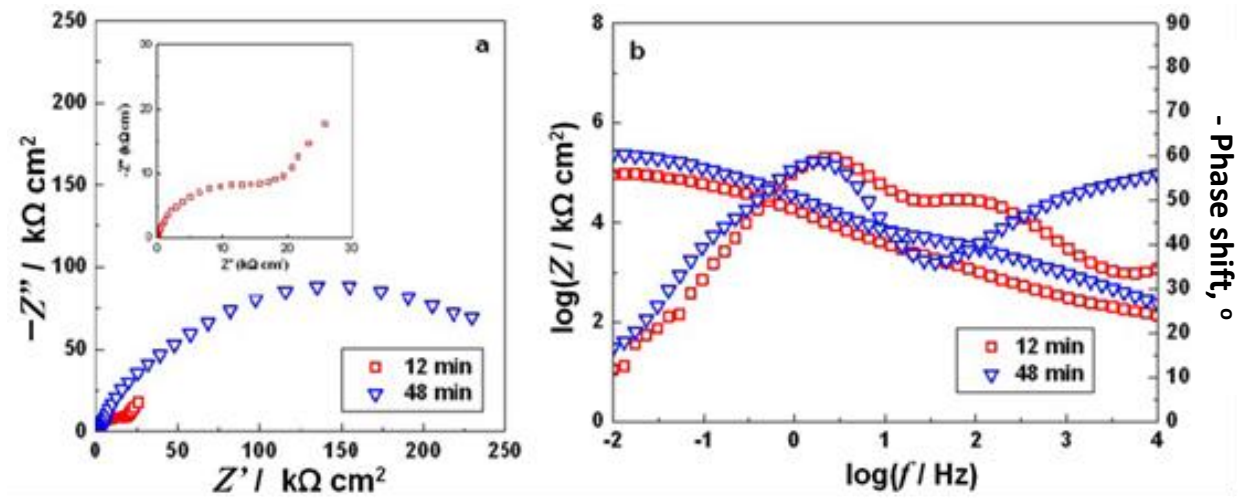

Figure 5. Averaged EIS spectra Bode plots of CeOPL deposited coatings after 12 and 48 minutes of anodization, acquired at the end of the exposition to the model corrosive medium

Both the real $\left(Z^{\prime}\right)$ and the imaginary $\left(-Z^{\prime \prime}\right)$ values of the smples anodized for 48 min are much superior to these of the samples with $12 \mathrm{~min}$ of anodization (Fig. 5a). The real impedance component reaches $250 \mathrm{k} \Omega \mathrm{cm}^{2}$ for the thicker AAO layer, whereas in the other case, its value is only $25 \mathrm{k} \Omega \mathrm{cm}^{2}$. Similar difference is observable for the logarithm of the total impedance in the respective Bode plot (Fig. 5b). The $\varphi(f)$-dependence recorded after 2,520 hours of exposure have two clearly distinguishable maxima, related to the AAO and CeOPL layers, at the lower and the higher frequency ranges. These maxima are almost completely overlapped in the EIS spectra acquired after (504/672 hours of exposure) of the samples anodized for $12 \mathrm{~min}$. This fact indicates that the coatings deposited after 48 min of anodization possess residual protective capabilities, although the interruption of their integrity after 2520 hours of exposure to the model $3.5 \% \mathrm{NaCl}$ corrosive medium.

The EIS results are further confirmed by the respective LVA measurements, performed after the sample exposure, shown in Fig. 6.
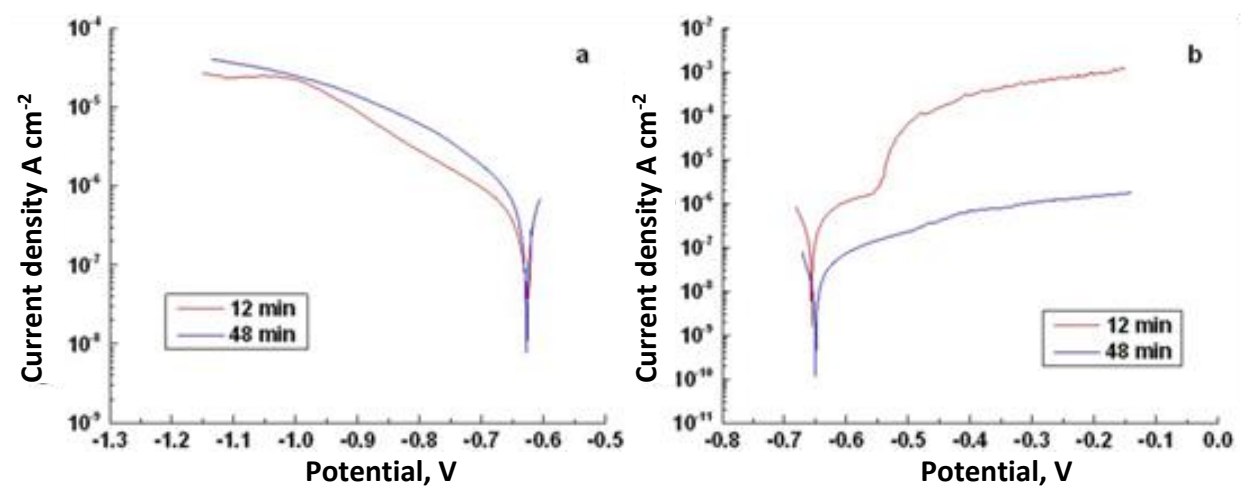

Figure 6. Cathodic (a) and anodic (b) polarization curves acquired at the end of the exposure of CeOPL coated samples after 12 and 48 minutes of anodization 
The cathodic polarization curves acquired after the corrosion tests for the specimens anodized for 12 and 48 min overlap, where there is obvious difference between the respective anodic curves. This fact is indication for the almost equal cathodic activity in the former case. However, there is a sharp current rise, in the anodic curve of the sample anodized for $12 \mathrm{~min}$, as a result of localized corrosion activity as is proposed elsewhere [30]. Consequently, the corrosion processes proceed with almost the same rate, but with a different mechanism.

Table 5. Fitting results for the anodized CeOPL coated specimens at the final exposure measurements

\begin{tabular}{|c|c|c|c|c|}
\hline \multicolumn{5}{|c|}{12 minutes of anodization } \\
\hline & & Sample 1S12 & Sample 2S12 & Sample $\mathbf{3 S 1 2}$ \\
\hline Element & Unit & After 504 hours & After 504 hours & After 672 hours \\
\hline$R_{\mathrm{el}}$ & $\Omega \mathrm{cm}^{2}$ & $14.18 \pm 0.20$ & $18.92 \pm 0.67$ & $57.48 \pm 16.28$ \\
\hline$Q_{\text {coat+oxy }}$ & $\mathrm{s}^{\mathrm{n}} \Omega^{-1} \mathrm{~cm}^{-2}$ & $0.68 \pm 0.01 \times 10^{-4}$ & $2.00 \pm 0.06 \times 10^{-5}$ & $1.44 \pm 0.09 \times 10^{-6}$ \\
\hline$n$ & & $0.86 \pm 0.03$ & $0.82 \pm 0.05$ & $0.66 \pm 0.06$ \\
\hline$R_{\text {coat+oxy }}$ & $\Omega \mathrm{cm}^{2}$ & $22.08 \pm 1.00 \times 10^{3}$ & $44.94 \pm 2.36 \times 10^{3}$ & $11.62 \pm 0.74 \times 10^{3}$ \\
\hline$Q_{\text {edl }}$ & $\mathrm{s}^{\mathrm{n}} \Omega^{-1} \mathrm{~cm}^{-2}$ & $0.51 \pm 22.96 \times 10^{-3}$ & $3.42 \pm 12.92 \times 10^{-4}$ & $0.78 \pm 0.08 \times 10^{-6}$ \\
\hline$n$ & & $0.95 \pm 0.09$ & $0.96 \pm 0.01$ & $0.92 \pm 0.02$ \\
\hline$R_{\mathrm{ct}}$ & $\Omega . \mathrm{cm}^{2}$ & $49.24 \pm 15.20 \times 10^{3}$ & $10.20 \pm 68.63 \times 10^{3}$ & $3.52 \pm 0.21 \times 10^{3}$ \\
\hline \multicolumn{5}{|c|}{48 minutes of anodization } \\
\hline & & Sample 1S48 & Sample $\mathbf{2 S 4 8}$ & Sample 3548 \\
\hline Element & Unit & After 2520 hours & After 2520 hours & After 2520 hours \\
\hline$R_{\mathrm{el}}$ & $\Omega \mathrm{cm}^{2}$ & $187.20 \pm 10.95$ & $85.60 \pm 10.46$ & $48.56 \pm 3.88$ \\
\hline$Q_{\text {coat+oxy }}$ & $\mathrm{s}^{\mathrm{n}} \Omega^{-1} \mathrm{~cm}^{-2}$ & $0.681 \pm 0.06 \times 10^{-5}$ & $3.22 \pm 0.09 \times 10^{-6}$ & $4.10 \pm 0.43 \times 10^{-5}$ \\
\hline $\mathrm{n}$ & & $0.65 \pm 0.01$ & $0.57 \pm 0.03$ & $0.69 \pm 0.01$ \\
\hline$R_{\text {coat+oxy }}$ & $\Omega \mathrm{cm}^{2}$ & $15.06 \pm 4.29 \times 10^{3}$ & $23.04 \pm 0.96 \times 10^{3}$ & $19.36 \pm 7.22 \times 10^{3}$ \\
\hline$Q_{\text {edl }}$ & $\mathrm{s}^{\mathrm{n}} \Omega^{-1} \mathrm{~cm}^{-2}$ & $0.81 \pm 0.43 \times 10^{-6}$ & $0.94 \pm 0.07 \times 10^{-6}$ & $2.37 \pm 1.08 \times 10^{-6}$ \\
\hline$n$ & & $1.00 \pm 0.11$ & $0.99 \pm 0.02$ & $1.00 \pm 1.48$ \\
\hline$R_{\mathrm{ct}}$ & $\Omega \mathrm{cm}^{2}$ & $21.84 \pm 11.40 \times 10^{5}$ & $12.30 \pm 0.27 \times 10^{5}$ & $71.60 \pm 6.29 \times 10^{4}$ \\
\hline
\end{tabular}

The anodic areas of the specimens, anodized for 48 min suffer uniform corrosion and the current density is distributed equally on the entire aluminum matrix, probably due to the initial galvanic corrosion stage. On the other hand, strong localized corrosion occurs for the samples anodized for $12 \mathrm{~min}$, after only 504/672 hours of exposure. This fact shows that the durability of the samples anodized for 48 min exceeds 2,520 hours, whereas the other samples (anodized for $12 \mathrm{~min}$ ) have already failed. All the polarization curves, recorded at the sample exposure termination, were submitted to further analysis and the obtained results for 12 and 48 min of anodization are summarized in Table 6.

Table 6. Tafel plot analysis of the polarization curves, recorded at the end of the exposure period

\begin{tabular}{ccccccccccccc}
\hline \multicolumn{1}{c}{ Cathodic } & \multicolumn{1}{c}{ Anodic } \\
\hline \hline $12 \mathrm{~min}$ & $1 \mathrm{~S} 12$ & $2 \mathrm{~S} 12$ & $3 \mathrm{~S} 12$ & $1 \mathrm{~S} 12$ & $2 \mathrm{~S} 12$ & $3 \mathrm{~S} 12$ & $1 \mathrm{~S} 12$ & $2 \mathrm{~S} 12$ & $3 \mathrm{~S} 12$ & $1 \mathrm{~S} 12$ & $2 \mathrm{~S} 12$ & $3 \mathrm{~S} 12$ \\
\hline \hline $504 \mathrm{~h}$ & -0.673 & -0.637 & - & 32.0 & 62.0 & - & -0.568 & -0.655 & - & 16.4 & 42.0 & - \\
$6,672 \mathrm{~h}$ & - & - & -0.641 & - & - & 17.8 & - & - & -0.699 & - & - & 1610.0 \\
\hline \hline $48 \mathrm{~min}$ & $1 \mathrm{~S} 48$ & $2 \mathrm{~S} 48$ & $3 \mathrm{~S} 48$ & $1 \mathrm{~S} 48$ & $2 \mathrm{~S} 48$ & $3 \mathrm{~S} 48$ & $1 \mathrm{~S} 48$ & $2 \mathrm{~S} 48$ & $3 \mathrm{~S} 48$ & $1 \mathrm{~S} 48$ & $2 \mathrm{~S} 48$ & $3 \mathrm{~S} 48$ \\
\hline \hline $2,520 \mathrm{~h}$ & -0.623 & -0.656 & -0.627 & 142.0 & 460.0 & 38.0 & -0.624 & -0.627 & -0.538 & 134.0 & 1380.0 & 960.0 \\
\hline
\end{tabular}

In both cases of the CeOPL coated samples anodized for 12 and $48 \mathrm{~min}$, the $R_{\mathrm{p}}$ values are by entire orders of magnitude higher than those of the reference samples, registered after 168 hours of exposure (Tables 4 and 6). Furthermore, the values of the samples anodized for 48 min are similar or slightly higher than those of the treated for only $12 \mathrm{~min}$. The $\mathrm{R}_{\mathrm{p}}$ values of the anodized specimens do not vary remarkably for the entire exposition period. Besides, this trend is in 
concordance with the EIS data fitting (Tables 3 and 5). All these facts indicate that both sets of anodized specimens possess residual barrier ability even after extended exposure periods. This conclusion was additionally confirmed by the morphological observations, which show weaker corrosion impact of the preliminary anodized samples.

Scanning Electron Microscopy and EDX-chemical analysis - The CeOPL coated AA2024-T3 specimens after 12, 24 and 48 min of anodization were submitted to systematic SEM observations before (Fig. 7) and after (Fig. 8) the corrosion tests, described in the previous sections.

The comparison among the positions in Fig. 7 reveal clear differences among the CeOPL layers, deposited after different anodization durations. Although the CeOPL layers were deposited, following the same procedure, these possess rather distinguishable morphologies, predetermined by the AAO underlayer formation process duration. Obviously (positions (a) and (b)), the AAO formed for only $12 \mathrm{~min}$ is disrupted over the alloys' intermetallic particles locations. That is the reason for the occurrence of brighter coarse particles in the locations of the coating disruptions. For comparison, such intermetallics were not observed in the other cases (24 and $48 \mathrm{~min}$ ). Another distinguishable feature observed in the case of $48 \mathrm{~min}$ of anodization (positions (c) and (f)) is the occurrence of net of cracks throughout the entire AAO/CeOPL layer surface.

This coating cracking is an indirect indication for a probably greater CeOPL layer thickness, achieved after $48 \mathrm{~min}$ of anodization. Besides, the cracks are not deep enough to cross the AAO underlayer, because these AAO/CeOPL conjunctions have shown remarkable durability, as was already commented. The concavities on the metallic surface observed for all the samples are result of the preliminary sample treatment procedures, as is discussed in previous works [31 - 33].

Similar systematic SEM observations have been done on the already corroded specimens. (i.e. CeOPL coatings after $0,12,24$, and 48 min of anodization and subsequent corrosion tests for 168 , 504,1008 and 2520 hours of exposure). The obtained SEM images are shown in Fig. 8.
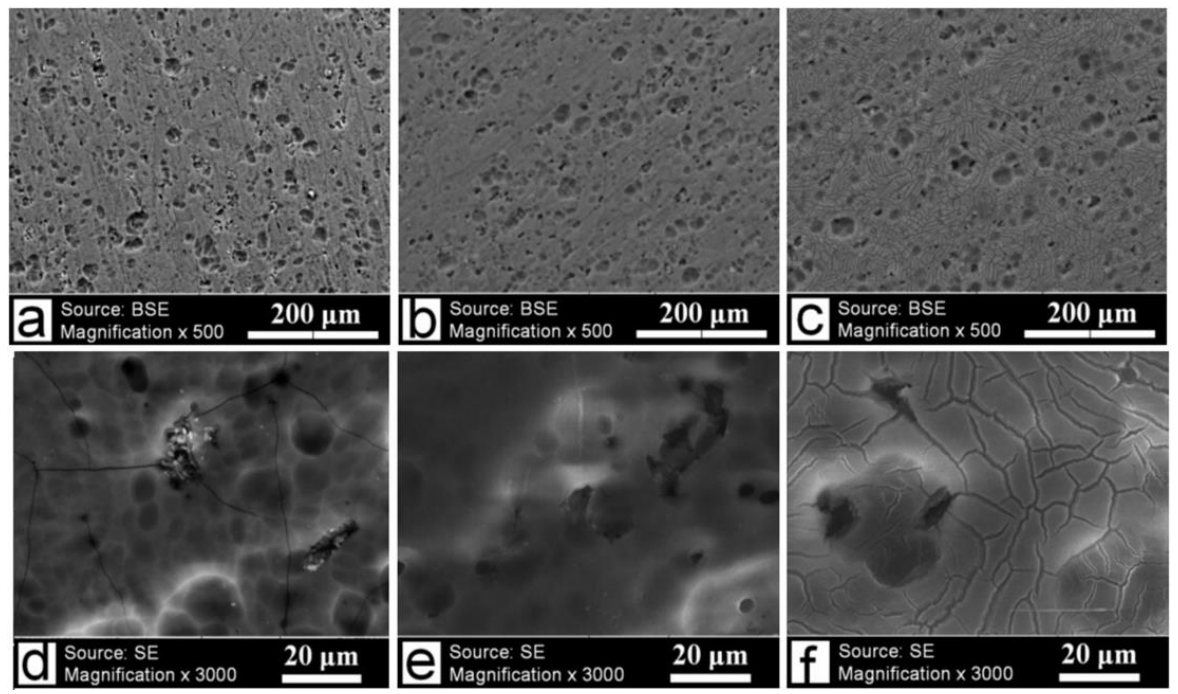

Figure 7. Low $(a, b, c)$ and high $(d, e, f)$ resolution SEM images of the investigated specimens after $12(a, d), 24(b, e)$ and $48(c, d)$ minutes of exposure

The SEM images in Fig. 8 clearly demonstrate the lack of corrosion pits on the sample, anodized for $48 \mathrm{~min}$, whereas in all the other cases occurrence of such pits was observed. Consequently, the $\mathrm{AAO} / \mathrm{CeOPL}$ combined layers coatings, formed at the largest anodization duration possess durability superior to 2,520 hours. In addition, these samples did not show any remarkable differences before and after the corrosion tests (Fig. 7 and 8). This fact is additional evidence for the superior sample durability. 

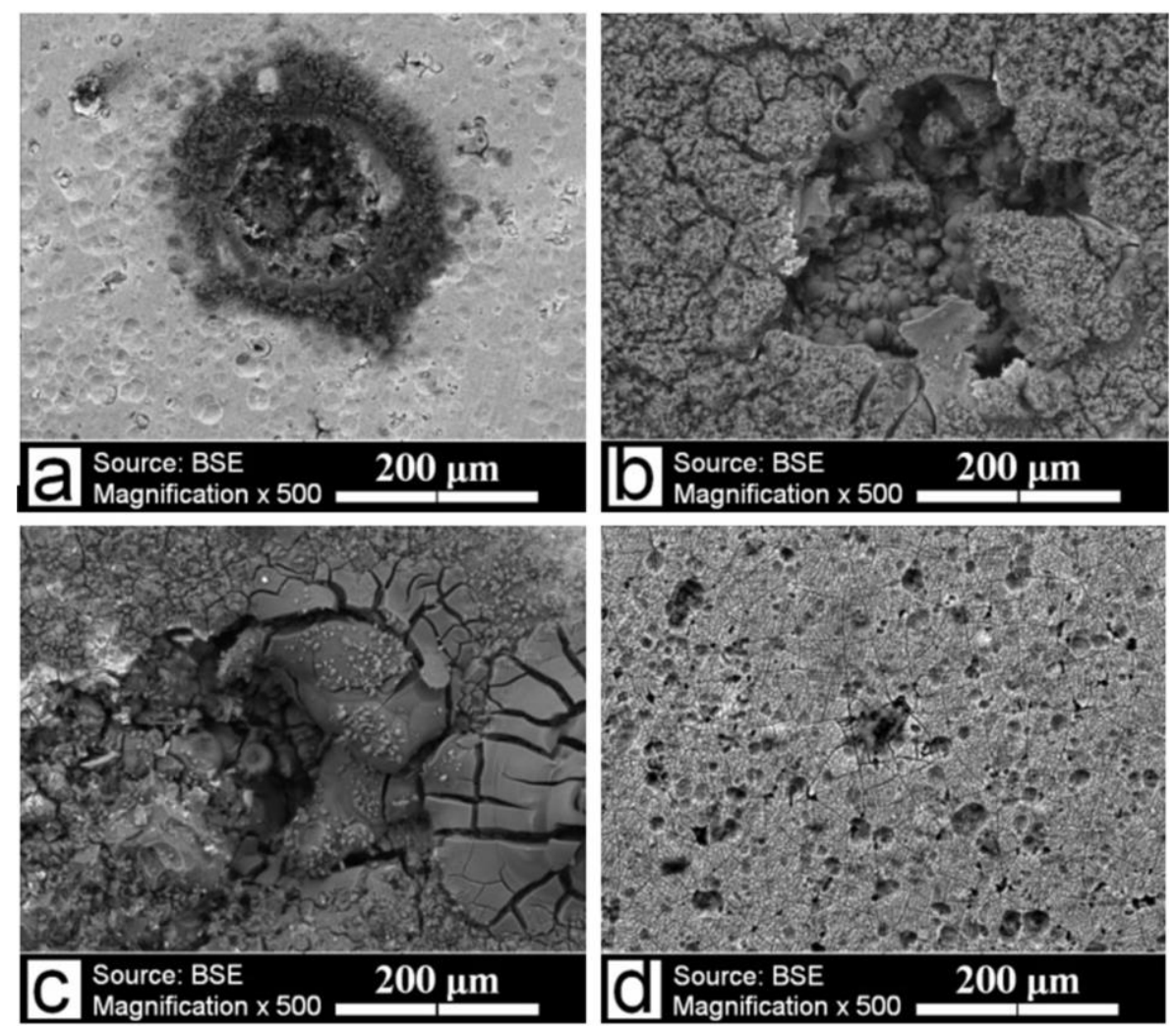

Figure 8. Low resolution SEM images of CeOPL coatings deposited after:

(a) $-0,(b)-12,(c)-24$ and (d) -48 minutes of anodization

The SEM observations were followed by EDX chemical analyses, in order to illustrate the coating chemical compositions, the elements distribution regularity, and the AAO/CeOPL coverage rate before the corrosion test procedures (Fig. 9 and 10).
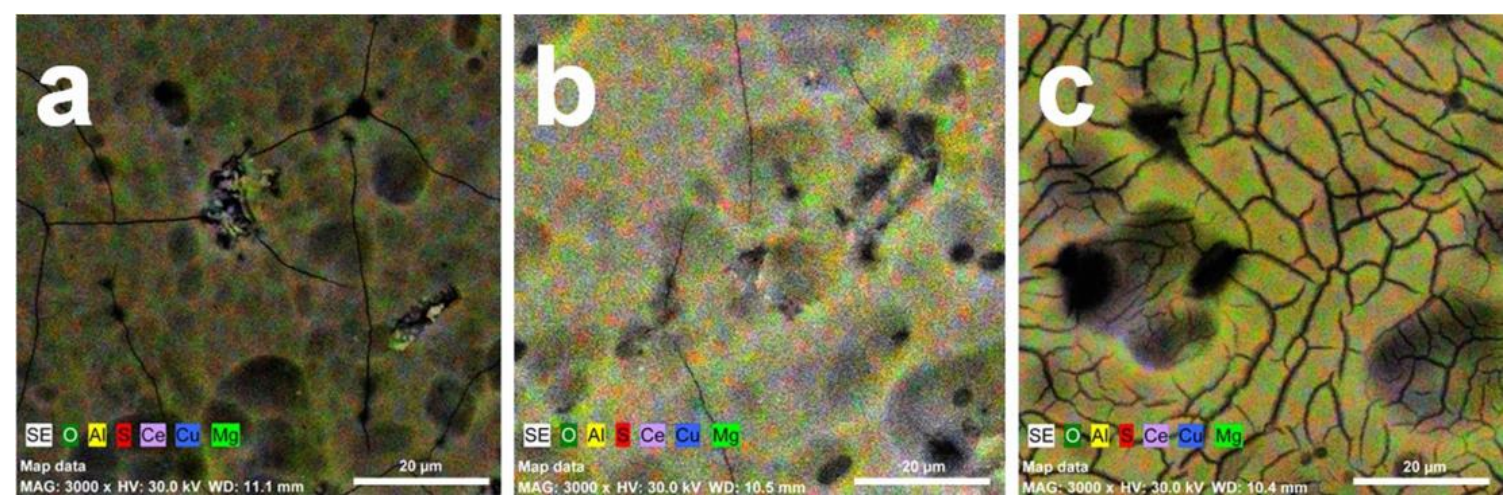

Figure 9. EDX map analyses of CeOPL coated AA2024-T3 samples, after (a) - 12, (b) -24 and (c) -48 minutes of anodization

The EDX map analyses reveal uniform element distribution, indicating uniform coverage of the metallic surface by the AAO and the further CeOPL layers. The basic alloy ( $\mathrm{Al}, \mathrm{Cu}$ and $\mathrm{Mg}$ ), AAO (Al, $\mathrm{S}$ and $\mathrm{O}$ ) and $\mathrm{CeOPL}$ (Ce and $\mathrm{O}$ ) elements were selected for observation.

The EDX images clearly reveal Mg re-deposition and sulfur entrapment in the AAO/ CeOPL layer composition. Unlike the results from previous works [8] the CeOPL layers deposited on the already anodized AA2024-T3 samples cover completely the metallic surface possessing dense structure and uniform element distribution (Fig. 10).

The SEM cross-sectional observations (Fig. 11) reveal gradual increase of the oxide layer thickness from 8 to $18 \mu \mathrm{m}$, within increasing of the anodization process duration from 12 to $48 \mathrm{~min}$, respectively. 

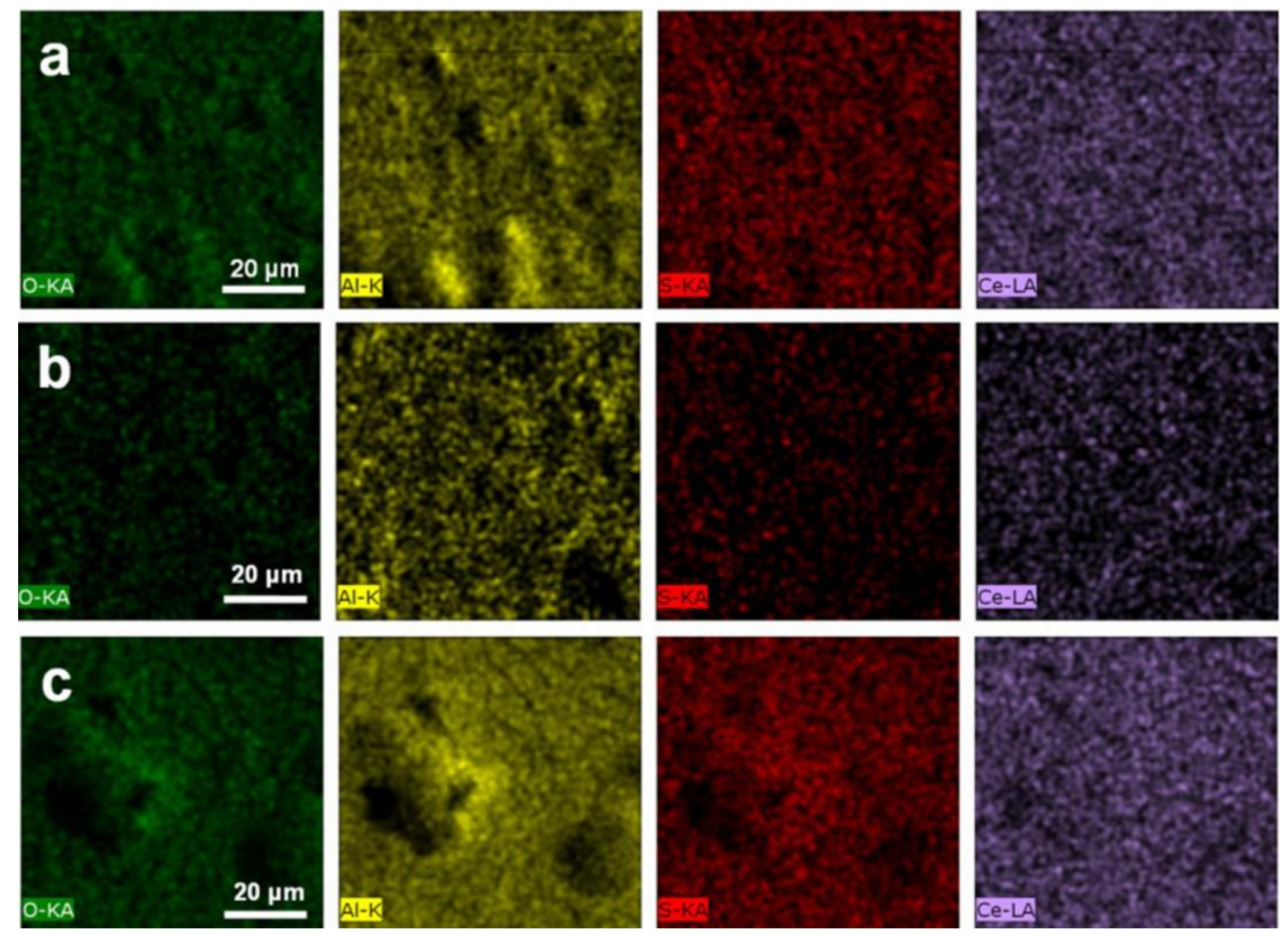

Figure 10. Element distribution of CeOPL coated AA2024-T3 samples, after (a) $-12,(b)-24$ and (c) -48 minutes of anodization

The correlation between the anodization process duration and the resulting AAO/ CeOPL layer thickness reveals an AAO thickness growth rate of about $600 \mathrm{~nm} \mathrm{~min}{ }^{-1}$. An interesting trend was established when the coating thickness and durability (Section 3.1) were compared.

For instance, the mathematical ratio between the durability test data $(504 \mathrm{~h})$ and the film

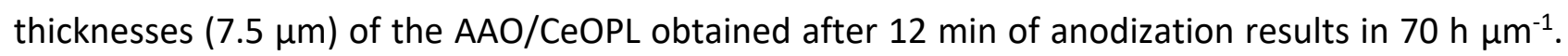
For the thickest film $(18.8 \mu \mathrm{m})$, obtained after $48 \mathrm{~min}$ of anodization with 2,520 h of durability this relation has $134 \mathrm{~h} \mathrm{\mu m}^{-1}$. This difference leads to the assumption that the anodization procedure proceeds with simultaneous film densification.

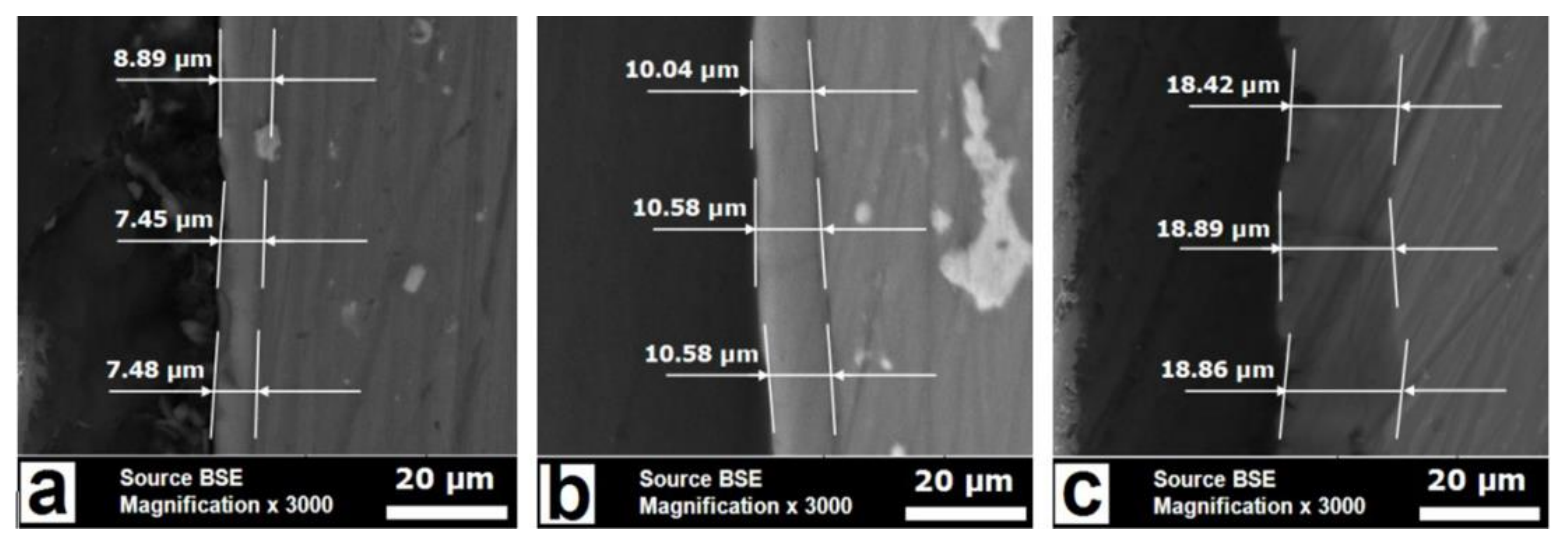

Figure 11. Cross-sectional SEM images of AAO/CeOPL double layered coatings deposited after:

$$
\text { (a) }-12 \text {, (b) }-24 \text { and (c) }-48 \text { minutes of anodization }
$$

Another interesting point is the element distribution inside the AAO/CeOPL film. The EDX map analyses reveal relatively equal cerium- and sulfur distributions across the investigated films (Fig. 12). 

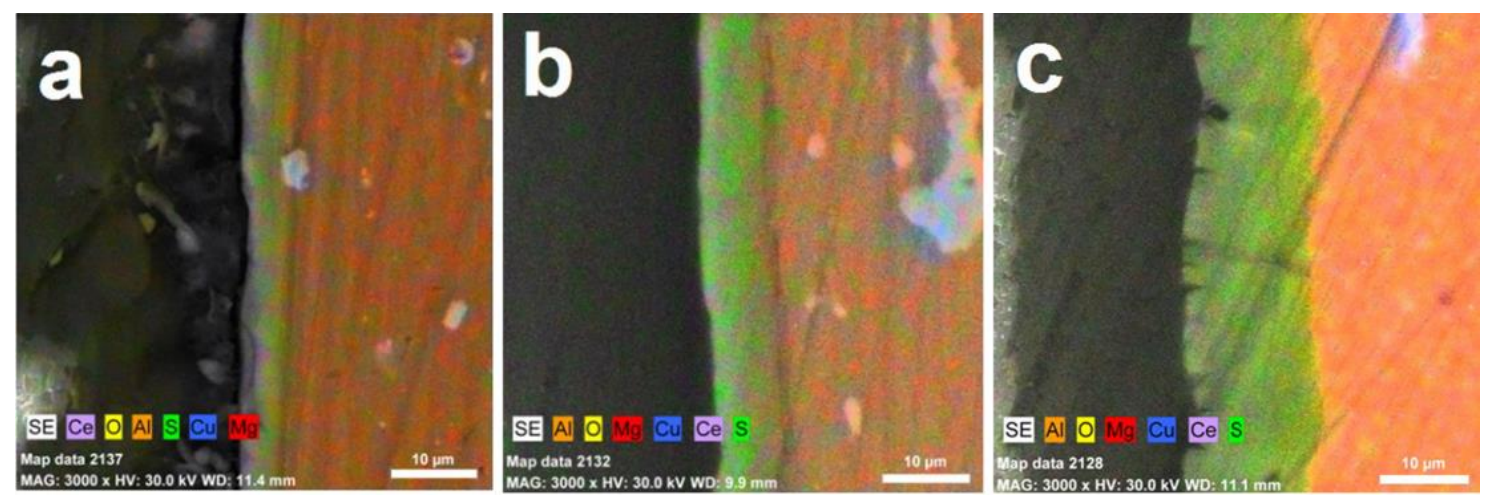

Figure 12. Cross-sectional EDX maps of AAO/CeOPL double layered coatings deposited after: (a) $-12,(b)-24$ and (c) -48 minutes of anodization

NSS-test procedures - The excellent performance of the AAO/CeOPL combined coating primers obtained by $48 \mathrm{~min}(18.8 \mu \mathrm{m})$ of anodization was undoubtedly confirmed by the NSS test procedures, because no corrosion pits were not registered after entire 168 hours of spraying with relatively concentrated $\mathrm{NaCl}$ solution.

\section{Conclusions}

The possibility for combination of Anodized Alumina Oxide (AAO) and Cerium Oxide Primer Layer (CeOPL) for elaboration of efficient protective coatings for AA2024-T3 aircraft alloy was proposed. The correlation between the anodization process duration (the resulting film thickness, respectively) and protective capabilities was evaluated by systematical characterization procedures. These procedures included electrochemical measurements (EIS, LVA), combined with morphological observations (SEM) and chemical element distribution (EDX). The protective properties of all investigated coatings were evaluated by extended exposure (up to 2,520 hours) to a $3.5 \% \mathrm{NaCl}$ model corrosive medium and regular electrochemical measurements. Additional cross-sectional SEM and EDX observations were performed in order to determine the AAO/ CeOPL film thickness (related to the anodization duration).

The obtained results regarding significant AAO/CeOPL layer durability were confirmed by standard neutral salt spray (NSS) test. The combination among these characterization methods has enabled to create complementary image for the coating properties and their performance. In addition, the correlations between the applied deposition procedures and the resulting $\mathrm{AAO} / \mathrm{CeOPL}$ coating layers were determined.

The measurement results have shown that the AAO/CeOPL conjunctions possess remarkable corrosion protective capabilities, unlike the reference CeOPL monolayers. The EIS spectra of the AAO/CeOPL conjunctions registered after 168 hours of exposure reveal much higher resistance values, compared to the referent CeOPL. Furthermore, the EIS spectra fitting required equivalent circuit for combined AAO/CeOPL layers, different from those for CeOPL reference coatings. This fact has revealed completely different performance in the model corrosive medium for both kinds of coatings. These conclusions from the EIS spectra analysis were confirmed by the respective polarization curves. The $R_{\mathrm{p}}$ values of the AAO/CeOPL layers were by entire orders of magnitude higher than those of the CeOPL references. The layers' durability increases progressively with the anodization duration. The specimens anodized for 12 minutes have resisted only about 600 hours, whereas $48 \mathrm{~min}$ of anodization has led to more than 2,520 hours of exposure until pitting appearance. The further data analysis, based on the film thickness determination has led to the inference that the coating durability increases progressively with the thickness growth (the ratio between the coating durability (600 to 2,520 hours) versus the film thickness (from 7.5 to $18.5 \mu \mathrm{m}$ ). 
The cross-sectional EDX map analyses have undoubtedly evinced the uniform distribution of the $\mathrm{AAO} / \mathrm{CeOPL}$ combined layer components (O, Al, S and $\mathrm{Ce}$ ).

The NSS test procedures have undoubtedly confirmed the excellent performance of the thickest AAO/CeOPL combined coatings (with $18.5 \mu \mathrm{m}$, obtained by $48 \mathrm{~min}$ of anodization).

Acknowledgements: The authors are grateful for the funding of this research to the Bulgarian National Scientific Research Fund, under contract NFNI: ДM-19/6 - 2017.

\section{References}

[1] W. J. Stepniowski, M. Moneta, K. Karczewski, M. Michalska-Domanska, T. Czujko, J.M.C. Mol, J. G. Buijnsters, J. Electroanal. Chem. 809 (2018) 59-66.

[2] S. Kozhukharov, C. Girginov, I. Avramova, M. Machkova, Mater. Chem. Phys. 180 (2016) 301-313.

[3] K. A. Yasakau, M. L. Zheludkevich, S. V. Lamaka, M. G. S, Ferreira, J. Phys. Chem. B 110 (2006) 55155528 ,

[4] G. Brunner, N. Birbilis, K. D. Ralston, S. Virtanen, Corros. Sci. 57 (2012) 209-214.

[5] K. D. Ralston, D. Fabijanic, N. Birbilis, Electrochim. Acta 56 (2011) 1729-1736.

[6] E. Kus, Z. Lee, S. Nutt, F. Mansfeld, Corrosion 62 (2006) 152-161.

[7] E. Sikora, X. J. Wei, B. A. Shaw, Corrosion 60 (2006) 387-398.

[8] S. Kozhukharov, M. Milanes, C. Girginov, M. Machkova, Mater. Corros. 67 (2016) 710-720.

[9] T. C. Tsai, T. H. Chuang, Mater. Sci. Eng. A 225 (1997) 135-144.

[10] A. E. Hughes, C. MacRae, N. Wilson, A. Torpy, H. T. Muster, A. M. Glenn, Surf. Interface Anal. 42 (2010) 334-338.

[11] J. van den Brand, W. G. Sloof, H. Terryn, J.H.W.de Wit, Surf. Interface Anal. 36 (2004) 81-88.

[12] E. Koroleva, G. Thompson, G Hollrigl, M Bloeck, Corros. Sci. 41 (1999) 1475-1495.

[13] S. S. Golru, M. M. Attar, B. Ramezanzadeh, Appl. Surf. Sci. 354 (2015) 360-368.

[14] S. Kozhukharov, Ch. Girginov, I. Avramova, M. Machkova, Mater. Chem. Phys. 180 (2016) 301-313.

[15] T. G. Harvey, Corros. Eng. Sci. Technol. 48 (2013) 248-269.

[16] M.J. O'Keefe, S. Geng, S. Joshi, Metalfinishing, 105 (2007) 25-28.

[17] V. Matolrın, M. Cabala, V. Chrab, I. Matolırnovar, K. Prince, M. Skoda, F. Sutara, T. Skarla, K. Veltruskra, Surf. Interface Anal. 40 (2008) 225-230.

[18] M. Škoda, M. Cabala, V. Cháb, K.C. Prince, L. Sedláček, T. Skála, F. Šutara, V. Matolín, Appl. Surf. Sci. 254 (2008) 4375-4379.

[19] I. Avramova, S. Suzer, D. Guergova, D. Stoychev, P. Stefanov, Thin Solid Films 536 (2013) 63-67.

[20] Y. Liu, J. Huang, J. B. Claypool, M. J. O'Keefe, J. Electrochem. Soc. 163 (2016) C198-C204.

[21] N. V. Skorodumova, S. I. Simak, B. I. Lundqvist, I. A. Abrikosov, B. Johansson, Phys. Rev. Lett. 89 (2002) 166601-166605.

[22] S. Kozhukharov, V. Kozhukharov, M. Schem, M. Wittmar, M. Veith, Prog. Org. Coat. 73 (2012) 95-103

[23] E. A. Matter, S. Kozhukharov, M. Machkova, V. Kozhukharov, Corros. Sci. 62 (2012) 22-33.

[24] E. A. Matter, S. Kozhukharov, M. Machkova, V. Kozhukharov, Mater. Corros. 64 (2013) 408-414

[25] M. Machkova, E.A. Matter. S. Kozhukharov, V. Kozhukharov, Corros. Sci. 69 (2013) 396-405.

[26] A. J. Aldykiewicz, A. J. Davenport, H. S. Isaacs, J. Electrochem. Soc. 143 (1996) 147-154.

[27] Ch. Girginov, S. Kozhukharov, M. Milanes, M. Machkova, Mater. Chem. Phys. 198 (2017) 137-144.

[28] M. A. Arenas, J. J. de Damborenea, Rev. Metal. Extra Vol. (2005) 433-437. (In Spanish)

[29] A. Conde, M.A. Arenas, A. de Frutos, J. de Damborenea, Electrochim. Acta 53 (2008) 7760-7768.

[30] M. Bethencourt, F. J. Botana, J. J. Calvino, M. Marcos, M. A. Rodriguez-Chacon, Corros. Sci. 40 (1998) 1803-1819

[31] E. A. Matter, S. Kozhukharov, M. Machkova, V. Kozhukharov J. Chem. Technol. Metall. 50 (2015) 52-64.

[32] E. A. Matter, S. V. Kozhukharov, M. S. Machkova, Bul. Chem. Commun. 43(1) (2011) 23-30.

[33] E. Matter, S. Kozhukharov, Ann. proceeds. Univ. Rousse (Bulgaria), 49 (2010) 14-19, acces via: http://conf.uni-ruse.bg/bg/docs/cp10/9.1/9.1-2.pdf

(C)2018 by the authors; licensee IAPC, Zagreb, Croatia. This article is an open-access article distributed under the terms and conditions of the Creative Commons Attribution license (http://creativecommons.org/licenses/by/4.0/) 